\title{
Feasibility study for development of a PET device based on Multi-gap Resistive Plate Chambers
}

\author{
M. Nizam ${ }^{1}$ \\ Tata Institute of Fundamental Research \\ E-mail: mohammad.nizam@tifr.res.in
}

\section{B. Satyanarayana, R.R. Shinde, G. Majumder}

Tata Institute of Fundamental Research

E-mail: bsn@tifr.res.in

Abstract: The Multigap Resistive Plate Chambers (MRPCs) provide excellent timing as well as position resolutions at relatively lower cost. Therefore, they can be used in medical imaging applications such as PET where precise timing is a crucial parameter of measurement. We have designed and fabricated several six-gap glass MRPCs and extensively studied their performance. In this paper, we describe the detector, electronics and the data acquisition system of the setup. We present here the data analysis procedure and initial results of our studies to measure the absolute position of a radioactive source ( $22 \mathrm{Na}$ ) using time of flight (TOF) as well as hit position information and hence to demonstrate their potential applications in medical imaging.

1.Introduction: The multigap resistive plate chamber (MRPC) is a gaseous detector which consist of several gas gaps sandwiched between two outer electrodes on which the high volatges are applied. MRPCs give excellent time and position resolution due to narrower subgaps compared to single gap RPCs. We have constructed several six-gap glass MRPCs of dimensions $305 \mathrm{~mm} \times 305 \mathrm{~mm} \times 7.5 \mathrm{~mm}$. The area of the internal glass plates are of dimension $256 \mathrm{~mm} \times 256 \mathrm{~mm} \times 0.410 \mathrm{~mm}$. Glass sheets of $2 \mathrm{~mm}$ thickness, coated with a conductive layer using graphite and paint of the NEROLAC brand, were used for the outer electrodes. The surface resistance of the conductive coat was in the range $(0.5-1)$ $\mathrm{M} \Omega / \#$. Two sided non conducting adhesive tapes were used on both sides of a mylar sheet to make small circular spacers of diameter $4 \mathrm{~mm}$ and thickness $\approx 250 \mu \mathrm{m}$. Twenty five spacers were used to maintain each gas gap. A detailed study of the detector characterization and it's performance can be found in [1]. We have made an attempt to demonstrate the feasibility of MRPC based PET imaging due to their excellent timing resulution.

2. Experimental Setup: We have mounted two six gaps glass MRPCs horizotally on top of each other separated by some distance which can be varied. The radioactive source is placed asymmtrically between the two detectors. A pair of scintillator paddles of dimensions $44 \mathrm{~cm} \times 44 \mathrm{~cm}$ are placed above the top MRPC and below the bottom MRPC such that the MRPCs are well within the area of scintillator paddles. The scintillator paddles are used to form a veto to reject cosmic muon background. We read only three $X$ and three $Y$ central strips of each MRPC to form the trigger. Three central strips $X$ plane and Three central strips of $\mathrm{Y}$ plane are ored separately and their oRed outputs are ANDed for each MRPC. The resultant AND output of each MRPC are finally ANDed to form the trigger. The AND signal of all four scintillator paddles is used as veto to remove the cosmic muon background. We have used only the $X$ plane timing data in this study. Figure 1 shows a photograph of the experimental setup and a schematic of the setup.

3. Time of Flight: The $X$ and $Y$ coordinates of hits are recorded along with the time of arrival of the photon at the detector. We obtain lines of response by joining the hit coordinates of MRPC1 and MRPC2 only for events with single multiplicity(with one strip hit per plane per detector). The timing Information infers the exact position of the source on the line of response.

The 39th International Conference on High Energy Physics (ICHEP2018)

4-11 July, 2018

Seoul, Korea

${ }^{1}$ Speaker 

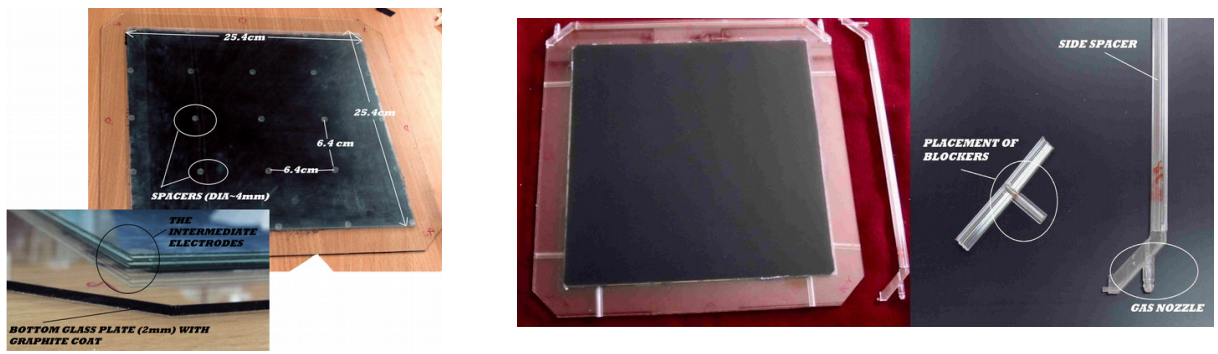

Figure 1. Fabrication of MRPC

The difference between timings of two opposite photons is calculated as $\Delta t=t_{\text {MRPC1 }}-$ $t_{\text {MRPC2}}$. There were mismatch in cable lengths and over all TDC path of different channels. To avoid this we have taken two reading for a fixed distnace between the MRPCs. First the source is kept at the bottom MRPC and we obtain $\Delta t_{1}=t_{M R P C 1}-t_{M R P C 2}$. The same reading is repeated but with source just below the top MRPC and we calculate $\Delta t_{2}=t_{\text {MRPC1 }}-t_{\text {MRPC2 }}$. . Finally we calculate the time of flight TOF=| $\Delta t_{1}-\Delta t_{2} \mid$. Our results of time of flight calculation has been summarized in Table 1.

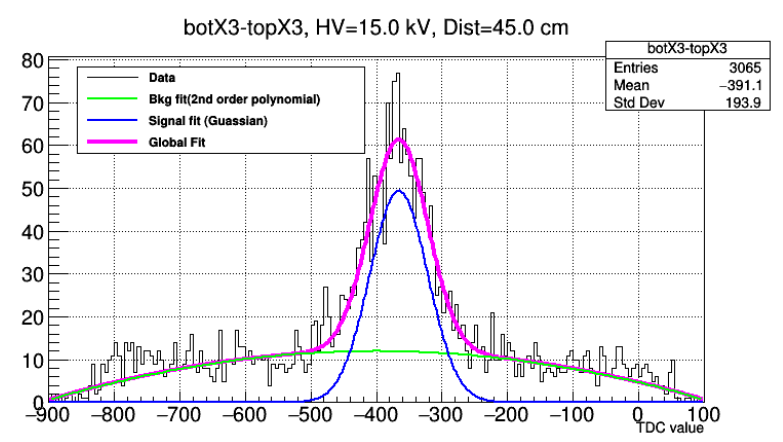

Figure 2. $\Delta$ t distribution at H.V. of $15 \mathrm{kV}$ and $\mathrm{d}=45 \mathrm{~cm}$

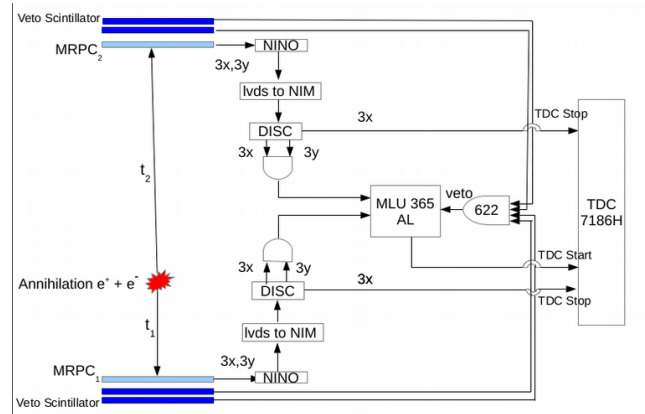

Block Diagram of the Setup

Figure 3. Setup with DAQ

\begin{tabular}{|c|c|c|c|c|c|}
\hline $\begin{array}{l}\text { Distance b/w } \\
\text { MRPCs }\end{array}$ & $\begin{array}{c}\text { Source at bottom } \\
\text { MRPC } \\
\Delta \mathrm{t}_{1} \\
\end{array}$ & $\begin{array}{c}\text { Source at top MRPC } \\
\Delta \mathrm{t}_{2}\end{array}$ & $\operatorname{diff}=\left|\Delta t_{1}-\Delta t_{2}\right| / 2$ & $\begin{array}{c}\text { Time of } \\
\text { flight(TOF) } \\
=\operatorname{diff} \times 25 \mathrm{ps}\end{array}$ & $\begin{array}{c}\text { Actual } \\
\text { TOF }\end{array}$ \\
\hline $30 \mathrm{~cm}$ & $\begin{array}{l}\text { Mean }=-335.8 \pm 2.1 \\
\text { Sigma }=54.76 \pm 2.4\end{array}$ & $\begin{array}{l}\text { Mean=-248.4 } \pm 2.8 \\
\text { Sigma=82.13 } \pm 3.5\end{array}$ & $43.7 \pm 2.4$ & $1.09 \pm 0.06 \mathrm{~ns}$ & $1.0 \mathrm{~ns}$ \\
\hline $45 \mathrm{~cm}$ & $\begin{array}{l}\text { Mean }=-369.4 \pm 2.3 \\
\text { Sigma }=61.57 \pm 2.7\end{array}$ & $\begin{array}{l}\text { Mean }=-256.8 \pm 2.1 \\
\text { Sigma }=59.56 \pm 2.1\end{array}$ & $56.3 \pm 2.2$ & $1.41 \pm 0.05 \mathrm{~ns}$ & $1.5 \mathrm{~ns}$ \\
\hline $60 \mathrm{~cm}$ & $\begin{array}{l}\text { Mean=-385.8 } \pm 2.8 \\
\text { Sigma=63.36 } \pm 3.6\end{array}$ & $\begin{array}{l}\text { Mean }=-223.5 \pm 2.4 \\
\text { Sigma }=-53.2 \pm 2.4\end{array}$ & $81.2 \pm 2.6$ & $2.03 \pm 0.07 \mathrm{~ns}$ & $2.0 \mathrm{~ns}$ \\
\hline $75 \mathrm{~cm}$ & $\begin{array}{l}\text { Mean }=-404.9 \pm 3.0 \\
\text { Sigma }=77.72 \pm 4.5\end{array}$ & $\begin{array}{l}\text { Mean }=-204.0 \pm 2.7 \\
\text { Sigma }=63.85 \pm 3.6\end{array}$ & \pm 2.8 & $2.51 \pm 0.07 \mathrm{~ns}$ & $2.5 \mathrm{~ns}$ \\
\hline
\end{tabular}

Table 1. Comaprison of the experimental and the expected TOF for various distances between MRPCs

4. Summary and Future Plan: The time of flight obtained from this study is in good agreement with the actual calculated values. The time resolution will improve after applying the time walk correction using pulse height information and applying correction for electronic jitter. We plan to get both analog and digital output from the front end preamplifiers to make time walk corrections. We will move to an FPGA based DAQ system designed for INO-ICAL experiment. MRPCs with converter material are under fabrication.

\section{References}

[1] M.M. Devi, N.K. Mondal, B. Satyanarayana, R.R. Shinde, Eur. Phys. J. C (2016)

[2] P. Fonte et al., [ALICE Collaboration] Nucl. Instrum. Meth. A 443, 201 (2000).

[3] M.S. Athar et al., [INO Collaboration], India-based Neutrino Observatory: Project Report. Volume I., INO-2006-01 